\title{
How Can Satellite DNA Divergence Cause Reproductive Isolation? Let Us Count the Chromosomal Ways
}

\author{
Patrick M. Ferree ${ }^{1}$ and Satyaki Prasad ${ }^{2}$ \\ ${ }^{1}$ W. M. Keck Science Department, The Claremont Colleges, Claremont, CA 91711, USA \\ ${ }^{2}$ Department of Molecular Biology and Genetics, Cornell University, Ithaca, NY 14853, USA
}

Correspondence should be addressed to Patrick M. Ferree, pferree@jsd.claremont.edu

Received 30 July 2011; Accepted 24 October 2011

Academic Editor: Vincent Sollars

Copyright (C) 2012 P. M. Ferree and S. Prasad. This is an open access article distributed under the Creative Commons Attribution License, which permits unrestricted use, distribution, and reproduction in any medium, provided the original work is properly cited.

\begin{abstract}
Satellites are one of the most enigmatic parts of the eukaryotic genome. These highly repetitive, noncoding sequences make up as much as half or more of the genomic content and are known to play essential roles in chromosome segregation during meiosis and mitosis, yet they evolve rapidly between closely related species. Research over the last several decades has revealed that satellite divergence can serve as a formidable reproductive barrier between sibling species. Here we highlight several key studies on Drosophila and other model organisms demonstrating deleterious effects of satellites and their rapid evolution on the structure and function of chromosomes in interspecies hybrids. These studies demonstrate that satellites can impact chromosomes at a number of different developmental stages and through distinct cellular mechanisms, including heterochromatin formation. These findings have important implications for how loci that cause postzygotic reproductive isolation are viewed.
\end{abstract}

\section{Introduction}

Decades ago when researchers began purifying DNA from eukaryotes using cesium chloride gradients, they observed bands of DNA that were distinct from the major genomic bands. The sequences comprising these ancillary bands were named satellites—a term from Greek meaning "followers of a superior entity" - and were found to separate from the other sequences due to their adenosine- and thymine-rich base pair compositions. Since their discovery, satellites have proven to be one of the most intriguing parts of the genome, owing to their high abundance, rapid evolutionary change, and a growing body of evidence indicating that they can impact speciation.

The abundance of satellites varies widely in eukaryotic genomes, from effectively $0 \%$ in yeast species such as Schizosaccharomyces pombe to $25-50 \%$ or more in Drosophila and mammalian species [2-4]. Individual satellite monomers also vary dramatically in their monomer length, from the D. melanogaster pentameric monomer, AATAT, to more complex monomers such as the 972-bp centromeric satellite in the Indian muntjac [5]. Satellite monomers such as these are organized into arrays, or blocks, of tens to thousands of tandem copies located in the centromeres, the telomeres, and their surrounding regions. Indeed, the $\mathrm{Y}$ chromosome in many higher eukaryotes consists almost entirely of satellites. Despite their abundance, satellites are nonprotein coding and were therefore hypothesized to be genomic "junk" [6] or even selfish genetic elements [7]. Contrary to the former idea, the chromosomal regions consisting of satellites are now known to play important but incompletely understood roles in the structure, stability, and segregation of the chromosomes [8-10]. The idea that satellites are selfish elements remains to be determined.

Given the high abundance of satellites and their involvement in chromosome behavior, it is intriguing that these sequences make up one of the most rapidly evolving parts of the genome. Studies conducted over the last four decades have revealed large disparities in satellite abundance between closely related species within insect, mammal, and plant groups [11-16]. Owing to rapid expansions and contractions in copy number, specific satellite blocks may be either severely reduced in size or altogether absent in close relatives (Figure 1) $[1,13,17,18]$. Additionally, the monomers of 


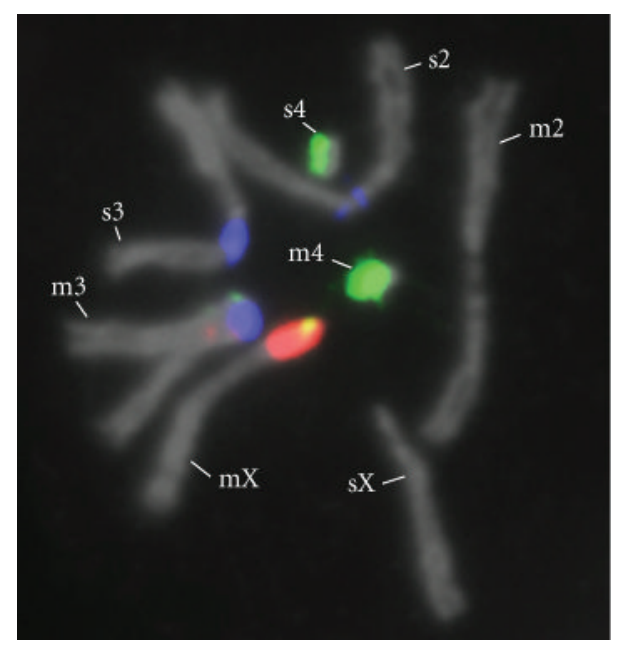

FIGURE 1: Satellite block divergence between Drosophila melanogaster and D. simulans. Each chromosome pair, consisting of one homologous chromosome from each species, shows remarkable satellite differences: the D. melanogaster X contains a large block of the 359bp satellite (red) and some AATAT (green) while the D. simulans X contains neither of these specific satellite monomers; dodeca satellite (blue) is present on the D. melanogaster 2nd chromosome and absent on the $D$. simulans 2 nd chromosome; large regions of dodeca satellite are present on the $3 \mathrm{rd}$ chromosomes of both species, but only D. melanogaster 3 rd chromosome has small regions of AATAT (green) and a small region of 359-bp variant (also red); AATAT satellite (green) is more abundant and distributed widely across the D. melanogaster 4 th chromosome while the D. simulans 4 th chromosome contains two primary regions of AATAT, which cannot be fully seen in this image, and in smaller amounts. Chromosomes were prepared from mitotic brain cells of hybrid larvae and stained by fluorescence in situ hybridization (FISH) as previously described [1].

some complex satellites can differ in sequence composition between closely related species at levels higher than the average genome-wide divergence [19]. However, certain regions of some centromere satellite monomers and even whole monomers are highly conserved, perhaps out of necessity to maintain their interactions with centromere-associated proteins [20-22].

Various mechanisms, including unequal recombination, gene conversion events, and replication slippage, have been proposed to explain how individual satellite blocks can evolve rapidly $[23,24]$. These processes can generate satellite blocks of widely varying sizes (i.e., those containing different copy numbers) within a given species. This variation can influence chromosome dynamics and individual fitness in a number of different ways. For example, large blocks of the D. melanogaster Responder ( $R s p$ ) satellite can be deleterious under certain genetic conditions. Located on the D. melanogaster 2nd chromosome, the Rsp block is highly variable, ranging from $\sim 10$ to over 3,000 monomers per block among individuals [25]. Second chromosomes carrying large Rsp blocks are targeted for destruction during spermatogenesis if the other 2nd chromosome carries a selfish allele of the Segregation Distorter $(S d)$ gene and a small Rsp block. This effect results in the loss of half the sperm-those carrying the large Rsp block-and, thus, high transmission frequencies of the $S d$-carrying chromosome. In contrast, variants of other satellite blocks may be functionally important for chromosome function and the fitness of the individual. One such case is the 359-bp satellite block on the X chromosome of $D$. melanogaster, which is located immediately adjacent to the rDNA locus and may play a role in regulating expression of the rDNA genes [26]. Finally, satellites can expand without affecting chromosome function. This trend appears to be true for satellites present on supernumerary B chromosomes, such as the Paternal Sex Ratio (PSR) chromosome in the jewel wasp, Nasonia vitripennis $[27,28]$. Since this chromosome is not essential for the viability of its host, the satellites on them may be free from functional constraints and, therefore, able to expand and contract rapidly without effect.

These observations raise a compelling question-how can rapid changes in satellites affect the biology of their resident chromosomes and, ultimately, the organisms in which they reside? One context in which this question can be addressed is the impact of satellite divergence on interspecies hybrids. Early studies demonstrated that certain reproductively isolated species - that is, those that fail to produce fertile or viable hybrid offspring when they intermate-can exhibit large differences in composition and organization of their satellite blocks $[1,11-14]$. These observations led to the suggestion that satellite divergence may contribute to speciation by causing reproductive isolation between species [11, 29]. Is there any validity to this idea, and if so, how might such an effect occur?

In addressing these questions, we describe three general ways in which satellite differences between species could affect chromosome behavior in hybrids: (i) by disruption of chromosome pairing, (ii) by alteration of the chromatin structure of the satellites themselves or their surrounding sequences, or (iii) by involvement of satellites in meiotic or postmeiotic chromosome drive systems. We cite data from previous studies, primarily in Drosophila but also other organisms, that either support or argue against these possibilities. We also describe plausible molecular mechanisms that may underlie these effects. These examples provide new ways of viewing the types of loci that cause reproductive isolation and how they can evolve and operate at the molecular level in hybrids.

\section{Disruption of Chromosome Pairing}

One process that satellite divergence may affect in hybrids is homolog pairing, whereby similar sequences associate together in close proximity across homologous chromosomes. Pairing is a key aspect of meiosis, and much of what is known about pairing during meiosis derives from studies in D. melanogaster. During meiosis I in this organism, pairs of homologous chromatids align side by side at the metaphase plate before they segregate into daughter nuclei. The pairing of homologous sequences occurs before entry into meiosis and is ultimately important in Drosophila and other eukaryotes across the phyla for proper segregation of 
chromosomes and, therefore, the formation of functional gametes [30].

There are, however, fundamental differences between male and female meiosis in flies that reflect to what degree satellite divergence may affect homolog pairing. In the pure species $D$. melanogaster, the involvement of repetitive sequences in pairing varies depending on the sex of the individual and the particular chromosome pair. For example, recombination occurs only in the female sex. Thus, synaptonemal complexes and chiasmata, or stable crossover junctions that help to hold the recombining homologs together before segregation, do not form in males [31]. The lack of these structures in males originally suggested that sequence specific interactions must instead dictate chromosome pairing in this sex $[32,33]$. Years of work on this topic have shown that small "pairing sites" mediate homolog pairing in males. These sites include sequences found in the gene-containing regions of the autosomes and a single cluster of rDNA spacer repeats on the $\mathrm{X}$ and $\mathrm{Y}$ chromosomes $[33,34]$. However, no data has been found to link satellite DNA or the pericentric regions where they are located with homolog pairing in male meiosis.

In contrast to male flies, satellites may play an important role in meiotic homolog pairing in female flies. Experiments in which recombination, and thus, chiasmata are prevented from forming either through mutations abrogating recombination or through chromosomal inversions revealed that pairing occurs without these structures (reviewed in [35]). Additionally, the 4th chromosomes are largely achiasmatic. Thus, pairing in females is determined not by recombination-mediated structures but instead by sequence-specific interactions. Deletions of the satellite-containing $\mathrm{X}$ and 4 th pericentric regions, but not the gene-containing regions, were shown to disrupt meiotic homolog pairing in females [35]. Thus, unlike in males, pericentric repetitive sequences may play a strong role in homolog pairing in females.

The fact that the pericentric regions do not influence homolog pairing in pure species $D$. melanogaster males leads to the strong expectation that interspecies divergence of satellite DNA would not affect pairing in Drosophila hybrid males. However, the involvement of these regions in female meiosis legitimizes early speculation that substantial differences in satellites may inhibit meiotic homolog pairing in Drosophila hybrid females [29]. Is there any experimental evidence for these predictions? D. melanogaster/D. simulans hybrids of either sex normally do not produce gonads, thus precluding the analysis of homolog pairing in these individuals. In order to circumvent this problem, partial male hybrids - those carrying small chromosomal regions or single chromosomes from one species in the genetic background of the other species-were produced [36]. Of particular interest was one type of partial male hybrid containing both the D. melanogaster and D. simulans 4th chromosomes. These interspecific homologs were found to pair and segregate normally during meiosis [36] despite substantial differences in their satellite DNA content [13]. This result is consistent with the lack of involvement of repetitive sequences in meiotic homolog pairing in D. melanogaster pure species males.
Currently, only a few other animal and plant hybrids have been examined. These analyses have focused primarily on the male sex, and while mispairing has been observed in some cases, the findings generally do not support a role of satellite divergence as a cause. In mice, male hybrids produced from Mus musculus and M. poschiavinus showed normal homolog pairing despite substantial, genome-wide differences in repetitive sequences [37]. In another case, $M$. domesticus/ M. spretus male hybrids exhibited defective X-Y pairing [38]. The causal locus was mapped to a region near the cytological point of pairing between these chromosomes in the pure species. This finding suggested that a single pairing site, similar to the one that determines pairing of the $\mathrm{X}$ and $\mathrm{Y}$ in D. melanogaster males, is solely involved. In plants, crosses between species belonging to the Paeonia genus revealed incomplete homolog pairing in several different species combinations [39]. Because no major chromosomal inversions were found between these species, it was concluded that mispairing likely resulted from interspecies divergence of pairing genes. However, divergence of repetitive sequences was not discussed as formal possibility.

Taken together, the above results suggest that satellite divergence does not affect meiotic homolog pairing in hybrids under certain species-, sex-, and chromosome-specific contexts. However, additional experiments are needed in other contexts, such as $\mathrm{X}$ or 4 th homolog pairing in Drosophila hybrid females, in which there is a strong precedence for expecting such an effect. Studies employing specific mutations that allow D. melanogaster/D. simulans hybrid females to develop functional gonads $[40,41]$ will be helpful in more fully addressing the impact of satellite divergence on meiotic homolog pairing.

Homolog pairing also occurs in the somatic tissues of Dipterans [42]. It has been proposed that somatic homolog pairing may play a role in the repair of double strand DNA breaks, the transitioning of premeiotic cells into meiosis, or transchromosome gene interactions [34, 42, 43]. Similar to meiotic pairing in females, pairing in somatic cells occurs between the pericentric regions in D. melanogaster [44]. What drives these interactions is not clear, but one possibility is high similarity of repetitive sequences between homologous chromosomes. This idea was argued against, however, by the results of one study in which a $\sim 1.6$ megabase pair block of AAGAG satellite located on the tip of the rearranged D. melanogaster 2 nd chromosome, $b w^{\mathrm{D}}$, was recombined onto the $D$. simulans 2 nd chromosome and placed into the $D$. simulans genome [45]. In the D. melanogaster pure species, this satellite block associated with the pericentric region of the same 2 nd chromosome, which also contains several blocks of AAGAG. When placed into the D. simulans genome, the $b w^{\mathrm{D}}$-derived AAGAG block associated with the pericentric region on the 2 nd chromosome of this species, despite the fact that it does not contain AAGAG satellite DNA. Moreover, the $b w^{\mathrm{D}}$-derived AAGAG block did not associate with either of the $D$. simulans sex chromosomes, which do contain AAGAG satellite DNA. It was concluded from these results that pairing in somatic cells might not result from similarity of homologous sequences, but instead, 
through sequence-independent attractive forces between large regions of repetitive DNA.

This conclusion may only partially explain somatic homolog pairing. Sequence-independent pairing alone would be expected to result in inappropriate associations of nonhomologous chromosomes during mitosis, and their missegregation, since all chromosomes in flies contain large amounts of repetitive sequences in their pericentric regions $[11,13]$. A more likely scenario may be that both sequence-dependent and independent interactions govern pairing in somatic cells. Previous experiments have demonstrated that somatic pairing in the D. melanogaster pure species occurs at specific pericentric regions, such as the Rsp locus as well as AACAC and AAGAC satellite blocks [44]. Interestingly, the Rsp block is not present on the 2nd chromosome in D. simulans [46], and other pairing sequences may also be unique or substantially different between these species. Thus, the D. simulans/ $D$. melanogaster hybrid is a promising system for taking advantage of these satellite differences in order to more fully explore the effects of satellite divergence on somatic homolog pairing.

\section{Alteration of Chromatin Structure I: Satellite DNA/Protein Interactions}

Another fundamental aspect of chromosome dynamics is the formation of chromosomes from chromatin. Occurring at entry into mitosis and meiosis, this process involves a number of structural proteins including Condensins and Topoisomerases [47]. These factors become distributed across the entire axes of the chromosomes as they condense at prophase. Other proteins, however, localize to discrete chromosomal regions, such as satellite blocks. For example, the D. melanogaster GAGA factor binds to AAGAG and AAGAGAG satellite monomers located in discrete regions on all of the chromosomes in this species [46]. GAGA factor and other satellite-binding proteins, such as Prod, are also transcription factors [48, 49].

The nature of these satellite DNA/protein associations is not well understood. However, it has been proposed that satellite-binding transcription factors may play a role in bending or packaging satellite DNA $[26,50,51]$. This idea is supported by the observation that loss-of-function mutations in the gene encoding GAGA factor result in severe chromosome decondensation and segregation failure [52]. Additionally, this result is consistent with the fact that GAGA associates with the FACT complex, which together may play a more global role in chromatin packaging of repetitive sequences [53].

A potential effect of satellite divergence is that it can drive coevolutionary changes in satellite-binding proteins within the pure species $[21,54]$. According to this model, the sets of satellites and their binding proteins will evolve independently from those of different species. A consequence of these independent evolutionary trajectories is that a diverged protein from one species may not properly bind a satellite variant of another species in the hybrid background. This loss-of-function effect may occur particularly in cases in which satellite-binding proteins from only one parental species are expressed in hybrids, such as proteins encoded by X-linked genes in hemizygous males or proteins that are maternally contributed in the egg cytoplasm. Similar effects might also be expected to result in cases where a protein from one species is expressed at low levels or not at all so that satellite DNA is insufficiently packaged. Such a case has not yet been demonstrated in hybrids, but is a formal possibility and might resemble chromatin defects caused by mutational loss of GAGA factor in D. melanogaster [52]. Alternatively, deleterious gain-of-function interactions may occur, such as if a satellite-binding protein from one species associates inappropriately either with a diverged or functionally unrelated satellite or with a chromatin-modifying enzyme of another species.

Compelling evidence of a satellite DNA/protein incompatibility was revealed through studies of the Odysseus-site homeobox $(\mathrm{OdsH})$ protein in Drosophila hybrids. Crosses between $D$. simulans males and D. mauritiana females produce F1 hybrid males that are sterile. Interspecies cloning strategies identified D. mauritiana OdsH (OdsHmau), located on the $\mathrm{X}$ chromosome of this species, as a causal locus [55]. Although its function is unknown, OdsH is homologous to Unc-4, a known transcription factor, and is expressed in the apical end of the testes where the mitotic divisions preceding meiosis occur $[56,57]$. Transgenic analysis revealed functional divergence between OdsH orthologs and the satellite DNA sequences to which it binds in each of these species. When expressed transgenically in $D$. simulans cells, OdsHsim and OdsHmau associated with similar satellite DNA regions on the $\mathrm{X}$ and 4 th chromosomes [58]. However, OdsHmau bound to many additional regions on the D. simulans $\mathrm{Y}$ chromosome [58]. The specific amino acid changes between OdsH orthologs that give rise to their different binding patterns are not known, although substantial sequence divergence was discovered in the OdsH DNA-binding homeodomain [55]. OdsHmau recognizes only a small region of satellite DNA on the D. mauritiana Y-chromosome, suggesting that the sequences to which it binds have undergone expansion across the D. simulans $\mathrm{Y}$ chromosome [58]. Thus, interspecies divergence of both $\mathrm{OdsH}$ and its associated satellite DNAs appears to underlie these different binding patterns between $D$. simulans and D. mauritiana.

It is currently unclear if hybrid sterility in this case results directly from differential OdsH binding to $\mathrm{Y}$ chromatin or to malfunction of an additional role of OdsH in the male germ line. However, several observations support the former possibility. First, deletion of the OdsH gene in D. melanogaster has little or no measurable effects on male fertility, demonstrating that OdsH is not an essential gene [56]. Second, the D. simulans $\mathrm{Y}$ becomes abnormally de-condensed in the presence of OdsHmau [58]. This effect could prevent the other chromosomes from segregating properly in the divisions preceding meiosis, thus leading to improper formation of sperm.

How might OdsHmau induce Y decondensation? One possibility is that this protein may bind satellites on the $D$. simulans $\mathrm{Y}$ that it normally binds on the $D$ mauritiana $\mathrm{Y}$, but expansion of these sequences in the former species may lead 
to a chromosomal overloading of OdsHmau. Alternatively, OdsHmau may associate with expanded sequences on the D. simulans $\mathrm{Y}$ that are distinct from those that it normally binds in D. mauritiana. In either case, high concentrations of OdsHmau may disrupt normal localization of other essential chromatin proteins. Identification of OdsH polymorphisms that cause differential DNA binding, and the specific satellite DNA sequences and other chromatin proteins that OdsH interacts with in each species, will be helpful in exploring these possibilities.

\section{Alteration of Chromatin Structure II: Heterochromatin-Related Effects}

Another potential effect of satellite divergence in hybrids is disruption of heterochromatin. This term describes the exceptionally dense form of chromatin that packages satellites and other highly repetitive sequences during interphase (for a full review, see [59]). Two primary molecular features that define heterochromatin and govern its compact nature are (i) specific posttranslational Histone modifications and (ii) a small set of associating non-Histone proteins. The basic unit of chromatin is the nucleosome, consisting of DNA wrapped around an octamer of the Histone proteins $\mathrm{H} 2 \mathrm{~A}, \mathrm{H} 2 \mathrm{~B}, \mathrm{H} 3$, and H4. In heterochromatin, the C-terminal "tail" of Histone $\mathrm{H} 3$ carries methyl groups on Lysine residues 9 and 27. Added by Histone Methyltransferases (HMTs), these methyl groups serve as binding sites for non-Histone proteins such as the heterochromatin protein 1 (HP1) and its protein family members $[60,61]$. It is believed that the association of HP1 with nucleosomes leads to the compact nature of heterochromatin $[62,63]$. In addition to binding methylated Histone H3, HP1 also binds SU(VAR)3-9, a HMT, thereby recruiting this enzyme to chromatin where it can insure methylation of Histone H3 [64, 65]. Thus, the interactions of these proteins with one another and with the nucleosomes constitute a selfregulatory system that maintains the heterochromatic state, which can be epigenetically transmitted through cell lineages.

Support for the idea that satellite DNA divergence can disrupt heterochromatin stems from studies of the D. melanogaster Zygotic hybrid rescue (Zhr) locus. Crosses between wild type $D$. melanogaster males and $D$. simulans females produce hybrid daughters that die during the cleavage divisions of early embryogenesis [66]. Previous genetic studies mapped a causal locus, $Z h r$, to a position near the centromere of the D. melanogaster X-chromosome [67]. Based on these and other genetic experiments [68, 69], it was proposed that $Z h r$ consists of repetitive sequences in this region, a novel idea given that many of the known loci involved in reproductive isolation are protein-coding genes $[55,70-$ 72]. More recent cytological analyses have supported this idea, demonstrating the presence of highly stretched region of 359-bp satellite DNA located on the D. melanogaster X during anaphase of mitosis in dying hybrid embryos [1]. This satellite region was found to prevent separation of the D. melanogaster sister X chromatids, inducing chromosome bridges and mitotic arrest (Figure 2).

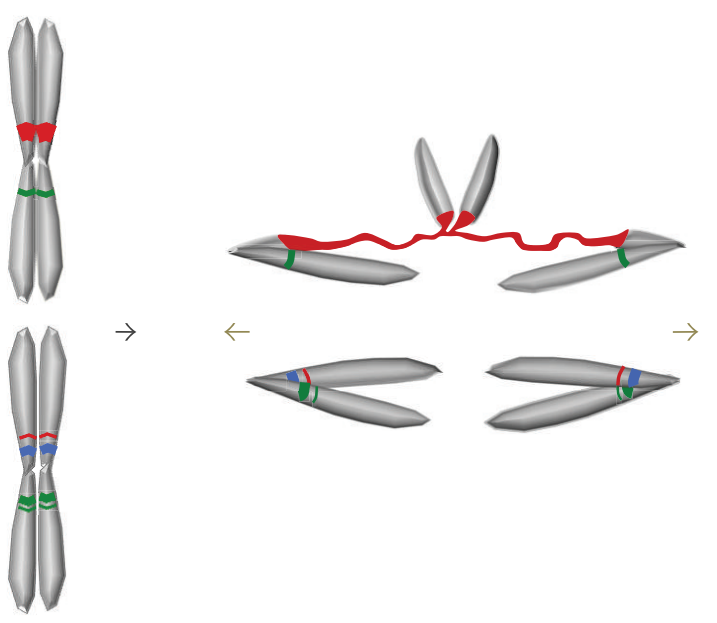

FIGURE 2: Disruption of mitotic chromosome segregation in hybrid embryos caused by satellite chromatin defects. Chromatid pairs line up at the metaphase plate for segregation at anaphase (left of arrow). The top chromatids fail to segregate due to defective chromatin structure of the red satellite block (right of arrow). This phenotype is analogous to that involving the 359-bp satellite block in D. melanogaster/D. simulans hybrid embryos [1] and results from an incompatibility between a $D$. melanogaster-specific satellite and a putative chromatin-related factor in the $D$. simulans egg cytoplasm.

Two specific findings support the idea that these defects are due to improper heterochromatin formation. First, Topoisomerase 2 (Top2) was found to accumulate abnormally on the stretched 359-bp satellite block [1]. In addition to its enzymatic role in relieving supercoiled DNA, Top2 is a structural chromatin protein $[73,74]$. In D. melanogaster, this protein is normally enriched on 359-bp satellite DNA at interphase and becomes evenly distributed across the chromosomes during mitosis [1]. In hybrids, however, Top2 remains abnormally localized to 359-bp satellite DNA throughout the cell cycle [1]. It is unlikely that D. simulans Top2, which is the only form present in the hybrid maternal cytoplasm, is the proximal cause, since this protein is highly conserved between D. melanogaster and D. simulans [1]. Moreover, hybrid females of the reciprocal cross are fully viable. Although only D. melanogaster Top2 is present in the egg cytoplasm of these individuals, D. simulans Top2 is expressed during later developmental stages while in the presence of the 359-bp satellite block, without deleterious effect.

Second, the observed chromosomal defects occur at the developmental period when heterochromatin forms. In Drosophila, heterochromatin formation is marked by visible changes in chromatin density during early embryogenesis. The first 14 rounds of mitosis in this organism occur in a common cytoplasm derived from the egg before the nuclei individualize through the acquisition of their own plasma membranes [75]. These early divisions proceed under the control of factors present in the maternal cytoplasm until the beginning of zygotic gene expression, which occurs during mitotic divisions 12-14. Heterochromatin formation is marked by the appearance of dense regions of chromatin known as chromocenters during mitotic divisions 9-10 
$[76,77]$. It is precisely during these divisions when the first chromosome bridges appear in hybrid female embryos [1].

Why might heterochromatin of the 359-bp satellite block fail to form in hybrids? One possibility is that some component(s) of the general heterochromatin machinery present in the $D$. simulans maternal cytoplasm are incapable of recognizing this D. melanogaster-specific satellite block. Although there is some precedence for this scenario in other systems [78], it is unlikely in this case for several reasons. First, the chromosome bridges in hybrid embryos appear during mitotic cycles 9-10, before HP1 and methylation of Histone H3 normally appear on the chromocenters [77]. Another general heterochromatin protein, SU(VAR)3-3, which is a homolog of the yeast demethylase LSD1, was recently shown to form foci in interphase nuclei as early as mitotic cycle 8, before bridge formation [79]. To our knowledge, however, this protein has not yet been examined for involvement in hybrid lethality. Second, the known protein components and posttranslational modifications to Histone $\mathrm{H} 3$ in heterochromatin, with few exceptions, are highly conserved from yeast to vertebrates [80]. This pattern stands in sharp contrast to the wide range of different satellite DNA sequences that exists within the genomes of most individual eukaryotic species, in all of which the heterochromatin machinery must properly package the entire sets of these sequences. It is, therefore, unlikely that the 359-bp satellite block poses challenges to the general heterochromatin machinery encoded by $D$. simulans.

An alternative explanation may involve small, noncoding RNAs. Studies in S. pombe demonstrated that small RNAs derived from centric and pericentric repeats and the proteins that produce these small RNAs are essential for normal heterochromatin structure and centromere function [81]. It was proposed that these small RNAs facilitate heterochromatin formation and maintenance by recruiting the heterochromatin machinery to their complementary sequences for proper packaging. Experimental evidence for this model has since been documented in a number of additional organisms including Arabidopsis thaliana and D. melanogaster [82-85]. Small RNAs derived from the 359-bp satellite have been detected in the maternal cytoplasm of young D. melanogaster embryos $[84,85]$. It was proposed that these small RNAs facilitate heterochromatin formation of the 359-bp satellite block in D. melanogaster [1, 82-84]. Moreover, the lack of the 359-bp small RNAs in the D. simulans-derived maternal cytoplasm of lethal hybrids may lead to mispackaging of this satellite block $[1,86]$. One appeal of this model is that it takes into account the specificity of the observed defects, which appear confined to the 359-bp satellite block; all other sequences in hybrids appear normally packaged [1]. The fact that only this satellite block exhibits packaging defects in hybrids may be due to its large size, comprising nearly one half of the pericentric region on the D. melanogaster X. Other satellite DNAs unique either to $D$. melanogaster or D. simulans may incur problems in heterochromatin packaging but they may not be present in enough copies to alter chromosome segregation.

Finally, the effects of 359-bp satellite DNA in hybrids may be tied to heterochromatin through parental imprinting.
Best studied in mammalian eukaryotes, imprinting is a phenomenon that results in differential expression of certain genes when inherited from either the mother or father. In Drosophila, parental imprinting does not affect protein-coding genes, but instead involves the heterochromatic regions of the X- and Y-chromosomes (reviewed in detail in [87]). Imprinting effects in flies include differential levels of silencing of visible genetic markers that are located near these particular regions of heterochromatin. For example, the scute gene, located near the pericentric heterochromatin of the inverted X chromosome, In (1) $s c^{8}$, is expressed at lower levels when paternally inherited compared to transmission from the mother $[88,89]$. Similar parental effects of reporter genes located within Y heterochromatin have also been observed $[90,91]$. The nature of heterochromatic imprinting is not understood but may involve sex-specific differences in $\mathrm{H} 3 \mathrm{~K} 9$ methylation of heterochromatin that are established during gamete formation and/or early development [87].

It is possible that the imprint of specific heterochromatic regions like the 359-bp satellite block may not be properly "interpreted" by the D. simulans maternal cytoplasm, resulting in the observed heterochromatin defects of this satellite in hybrids. One possible scenario is that the $D$. simulans cytoplasm fails to recognize $D$. melanogaster-specific Histone methylation or another unknown epigenetic mark on this satellite, which might be needed for proper heterochromatin packaging. Currently the Histone methylation state of the 359-bp heterochromatin has not been studied in hybrid embryos. However, a prediction based on the above hypothesis is that transmission of the 359-bp satellite block through the $D$. simulans maternal cytoplasm would result in suppression of packaging defects. Consistent with this prediction is the fact that hybrid females of the reciprocal cross, between $D$. melanogaster females and $D$. simulans males, are completely viable. In this case, the 359-bp satellite block should be imprinted maternally through the $D$. melanogaster egg cytoplasm. However, it is important to point out that the viability of reciprocal female hybrids is also consistent with mechanisms involving diverged satellite-binding proteins or repeat-derived small RNAs outlined above.

\section{Release of Meiotic and Postmeiotic Drive Systems}

Under normal circumstances, homologous chromosomes are segregated equally into gametes. However, some loci are capable of altering chromosome segregation during or after meiosis in order to selfishly transmit themselves at unusually high frequencies. In these cases, satellite variants can be either the targets of drive or the driving elements themselves (Figure 3).

One well-known example of postmeiotic drive involving satellites is the Segregation Distorter (SD) system in D. melanogaster. The selfish component of SD is a duplicated gene on chromosome 2 encoding a truncated RanGAP protein [92]. In males that are heterozygous for this mutant allele, $S d$, and the wild type allele, $S d^{+}$, the entire half of the spermatids containing the $S d^{+}$allele exhibit chromosome 


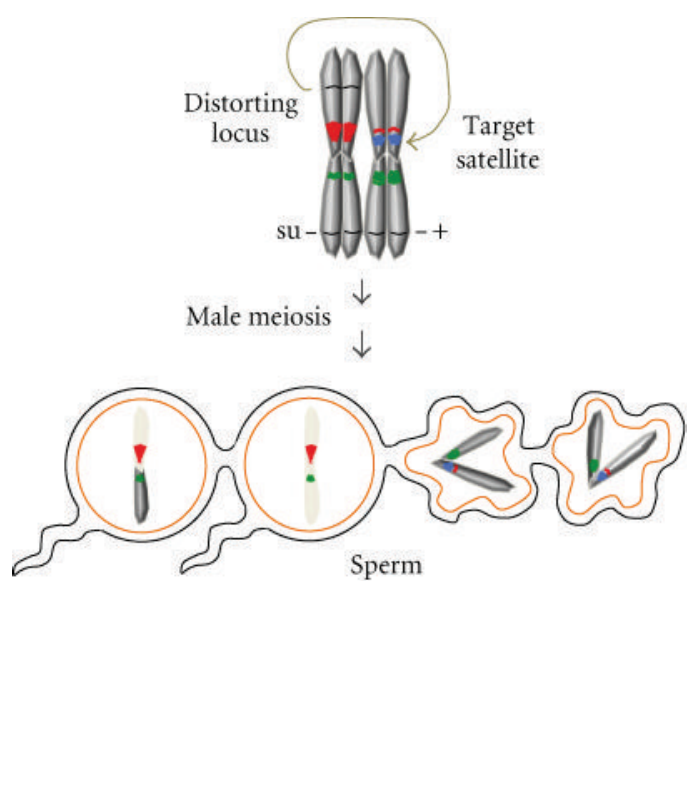

(a)

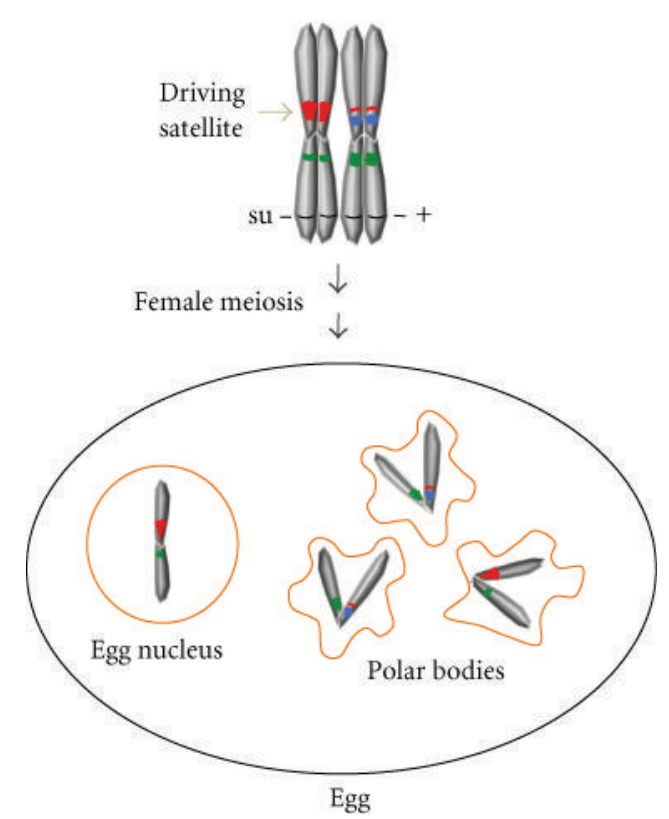

(b)

Figure 3: Segregation distortion in hybrid animals. (a) Postmeiotic release of segregation distortion in hybrid males. A recessive suppressor of distortion ( $\mathrm{su}$ ) in one species becomes inactive in the heterozygous hybrid. This allows the distorting locus to target a satellite block on the chromosomes of the other species (top). This effect results in spermatid bundles (bottom) in which spermatids inheriting the targeted chromosome fail to individualize. The spermatids carrying the chromosome with the distorting locus develop normally. (b) Release of meiotic drive in hybrid females. A recessive suppressor becomes heterozygous in the hybrid female. This enables a chromosome from one species, which carries a "selfish" satellite, to outcompete the homologous chromosome from the other species. As a result, the egg nucleus will carry a chromosome with the selfish satellite, and chromosomes lacking these satellites will end up in the unused polar bodies.

condensation defects and they fail to mature. Thus, only chromosomes carrying the selfish $S d$ allele are transmitted. $S d$ does not target the $S d^{+}$allele itself, but instead, a closely linked satellite block consisting of a 240-bp monomer known as Responder (Rsp). Rsp satellite blocks consisting of $\sim 200$ to 3,000 or more monomers (termed Responder-sensitive or $R s p^{\mathrm{S}}$ ) are targeted, whereas smaller blocks (Responder-insensitive or $R s p^{\mathrm{I}}$ ) are unaffected [25]. This effect favors $S d$ since it is linked to $R s p^{\mathrm{I}}$ blocks, whereas $S d^{+}$is often linked to $R s p^{S}$ blocks. It is currently not known how $S d$ targets $R s p^{S}$ satellite blocks at the molecular level, but may involve mislocalization of $S d$-encoded RanGAP that leads to chromosome decondensation through a number of possible mechanisms $[86,93,94]$.

Distorting loci like $S d$ may eventually harm individuals and populations, such as when distorters are closely linked to deleterious alleles, or if distortion involves the sex chromosomes, thus affecting the sex ratio balance in populations, respectively. As a counter, unlinked suppressors of distortion may evolve. Suppressors are effective until mating occurs with individuals that do not carry them, in which case suppression is lost and the driving phenotype is unleashed (Figure 3(a)). In agreement with this idea, several different masked distortion systems have been identified through both interstrain and interspecies Drosophila crosses [94, 95]. In these cases, the targets of distortion are not known, but may involve species-specific satellites since defects in spermatogenesis are highly similar to those present in Sd distortion [94].
Distorting loci can also be the satellites of centromeres or their adjacent regions. One process in which these sequences are thought to be particularly prone to non-Mendelian segregation is female meiosis. This is due primarily to the fact that meiosis in females is asymmetric; four meiotic products are produced but only one becomes the egg's hereditary material, while the other three products form polar bodies and are eliminated. It has been proposed that certain centromeric satellite variants can take advantage of this asymmetry by outcompeting other sequences for extraordinarily high rates of transmission into the egg's nuclear material (Figure 3(b)) [96-98].

Non-Mendelian segregation of certain alleles during female meiosis has been detected genetically in a number of organisms [99-102]. However, the most direct evidence for meiotic drive of repetitive elements stems from one study in Mimulus (monkeyflower) species hybrids. Crosses between Mimulus guttatus and M. nasutus resulted in release of a suppressed meiotic driver locus on the M. guttatus chromosome 2 that approaches transmission of $100 \%$ [103]. Genetic and cytological mapping revealed that the driving element is located in or immediately adjacent to the centromere, consistent with the possibility that the element is a satellite [102]. Interestingly, this driving allele is associated with a fitness cost in hybrid males. In the pure species, such deleterious effects may prevent selfish elements from reaching fixation before driving suppressors can evolve. Future molecular and cytological studies in this system will help to test existing 
models that predict how meiotic drive might occur at the molecular and cellular levels $[98,104]$.

\section{Satellite Divergence and the Dobzhansky-Bateson-Muller Model of Hybrid Incompatibility}

Early work by Dobzhanksy, Bateson, and Muller provided the foundation for a genetic model that explains the evolution of hybrid sterility and lethality [105]. The simplest form of this model involves a pair of loci, each of which has diverged functionally between sibling species. The products of these loci malfunction when expressed together in hybrids, leading to developmental defects that cause sterility or lethality. Such interspecies molecular interactions that reduce hybrid fitness are referred to as hybrid incompatibilities (HIs). Over the past decade, a number of HI loci have been identified. Some of these loci encode proteins [106]. It was proposed that HI loci encoding transcription factors cause large-scale misregulation of gene expression in $D$. simulans/ D. melanogaster hybrids [70], although this was later shown to not be the case [107]. Other models implicate deleterious interactions between proteins encoded by HI loci [108]. In general, much remains to be uncovered mechanistically regarding the majority of $\mathrm{HI}$ cases that involve protein-coding genes.

A number of studies discussed here have documented the negative effects of satellite divergence on chromosome behavior in hybrids. The results from these studies have demonstrated that satellites, like protein-coding genes, can operate as HI loci. The biology of satellites is complex, with a diverse array of associated factors including general and specific heterochromatin proteins, small RNAs, and epigenetically modified histones that are often developmentally regulated. This complexity offers researchers new ways to envision how $\mathrm{HI}$ might occur in hybrids and new HI candidates to test.

At the core of the evolution of such HI cases may be a scenario in which rapidly evolving satellite sequences force their packaging or associating proteins to evolve equally rapidly in order to preserve chromosome function in the pure species. However, proteins-or perhaps other factorsadapted to satellites from one species may interact inappropriately with diverged satellites from another species in hybrids, thus causing HI. The complex nature of satellite heterochromatin is consistent with previous speculation that most HI interactions may be more complex than the twolocus model [109]. Reciprocally, however, the existence of satellite HI loci may also offer more simplified views of $\mathrm{HI}$, such as an HI locus pair consisting of satellite DNA in one species and the absence of complementary small RNAs in the other species. Indeed, satellite DNA may even be regarded as a special type of $\mathrm{HI}$ locus because it can direct its own packaging by generating small RNAs, thus operating as both the cause and suppressor of $\mathrm{HI}[86]$.

Given the functional involvement of satellites in chromosome dynamics and their evolutionarily labile nature, it is no surprise that these sequences make up a common type of reproductive isolating locus. Further exploration will, no doubt, be challenging due to difficulties in manipulating sate-llite sequences and the epigenetic states of heterochromatin, but they will progressively reveal a more detailed picture of how these hybrid incompatibilities occur at the molecular level.

\section{Acknowledgments}

The authers would like to thank D. Barbash, V. Meller, and three anonymous reviewers for helpful comments.

\section{References}

[1] P. M. Ferree and D. A. Barbash, "Species-specific heterochromatin prevents mitotic chromosome segregation to cause hybrid lethality in Drosophila," PLoS Biology, vol. 7, no. 10, Article ID e1000234, 2009.

[2] F. T. Hacch and J. A. Mazrimas, "Fractionation and characterization of satellite DNAs of the kangaroo rat (Dipodomys ordii)," Nucleic Acids Research, vol. 1, no. 4, pp. 559-576, 1974.

[3] V. Wood, R. Gwilliam, M.-A. Rajandream et al., "The genome sequence of Schizosaccharomyces pombe," Nature, vol. 415, no. 6874 , pp. 871-880, 2002.

[4] G. Bosco, P. Campbell, J. T. Leiva-Neto, and T. A. Markow, "Analysis of Drosophila species genome size and satellite DNA content reveals significant differences among strains as well as between species," Genetics, vol. 177, no. 3, pp. 12771290, 2007.

[5] O. Vafa, R. D. Shelby, and K. F. Sullivan, "CENP-A associated complex satellite DNA in the kinetochore of the Indian muntjac," Chromosoma, vol. 108, no. 6, pp. 367-374, 1999.

[6] S. Ono, "So much "junk" DNA in our genome," Brookhaven Symposia in Biology, vol. 23, pp. 366-370, 1972.

[7] L. E. Orgel and H. C. Crick, "Selfish DNA: the ultimate parasite," Nature, vol. 284, no. 5757, pp. 604-607, 1980.

[8] G. H. Karpen, M. H. Le, and H. Le, "Centric heterochromatin and the efficiency of achiasmate disjunction in Drosophila female meiosis," Science, vol. 273, no. 5271, pp. 118-122, 1996.

[9] A. F. Dernburg, J. W. Sedat, and R. S. Hawley, "Direct evidence of a role for heterochromatin in meiotic chromosome segregation," Cell, vol. 86, no. 1, pp. 135-146, 1996.

[10] Y. Yamagishi, T. Sakuno, M. Shimura, and Y. Watanabe, "Heterochromatin links to centromeric protection by recruiting shugoshin,” Nature, vol. 455, no. 7210, pp. 251-255, 2008.

[11] M. Gatti, S. Pimpinelli, and G. Santini, "Characterization of Drosophila chromatin. I. Staining and decondensation with Hoechst 33258 and quinacrine," Chromosoma, vol. 57, no. 4, pp. 351-375, 1976.

[12] F. T. Hatch, A. J. Bodner, J. A. Mazrimas, and D. H. Moore, "Satellite DNA and cytogenetic evolution; DNA quality, satellite DNA and karyotypic variation in kangaroo rats (Genus Dipodomys)," Chromosoma, vol. 58, no. 2, pp. 155-168, 1976.

[13] A. R. Lohe and P. A. Roberts, "Evolution of satellite DNA sequences in Drosophila," in Heterochromatin: Molecular and Structural Aspects, R. S. Verma, Ed., Cambridge University Press, Cambridge, UK, 1988.

[14] A. Kamm, I. Galasso, T. Schmidt, and J. S. Heslop-Harrison, "Analysis of a repetitive DNA family from Arabidopsis arenosa and relationships between Arabidopsis species," Plant Molecular Biology, vol. 27, no. 5, pp. 853-862, 1995. 
[15] A. V. Vershinin, E. G. Alkhimova, and J. S. Heslop-Harrison, "Molecular diversification of tandemly organized DNA sequences and heterochromatic chromosome regions in some Triticeae species," Chromosome Research, vol. 4, no. 7, pp. 517-525, 1996.

[16] R. Ross, T. Hankeln, and E. R. Schmidt, "Complex evolution of tandem-repetitive DNA in the Chironomus thummi species group," Journal of Molecular Evolution, vol. 44, no. 3, pp. 321327, 1997.

[17] D. Ugarković, S. Durajlija, and M. Plohl, "Evolution of Tribolium madens (Insecta, Coleoptera) satellite DNA through DNA inversion and insertion," Journal of Molecular Evolution, vol. 42, no. 3, pp. 350-358, 1996.

[18] C. H. Slamovits, J. A. Cook, E. P. Lessa, and M. S. Rossi, "Recurrent amplifications and deletions of satellite DNA accompanied chromosomal diversification in South American tuco-tucos (genus Ctenomys, Rodentia: Octodontidae): a phylogenetic approach," Molecular Biology and Evolution, vol. 18, no. 9, pp. 1708-1719, 2001.

[19] T. Stratchan, D. Webb, and G. A. Dover, "Transition stages of molecular drive in multiple-copy DNA families in Drosophila," EMBO Journal, vol. 4, pp. 1701-1708, 1985.

[20] D. Kipling and P. E. Warburton, "Centromeres, CENP-B and Tigger too," Trends in Genetics, vol. 13, no. 4, pp. 141-145, 1997.

[21] H. S. Malik and S. Henikoff, "Adaptive evolution of Cid, a cen tromere-specific histone in Drosophila," Genetics, vol. 157, no. 3, pp. 1293-1298, 2001.

[22] N. Meštrović, P. Castagnone-Sereno, and M. Plohl, "Interplay of selective pressure and stochastic events directs evolution of the MEL172 satellite DNA library in root-knot nematodes," Molecular Biology and Evolution, vol. 23, no. 12, pp. 23162325, 2006.

[23] B. Charlesworth, P. Sniegowski, and W. Stephan, "The evolutionary dynamics of repetitive DNA in eukaryotes," Nature, vol. 371, no. 6494, pp. 215-220, 1994.

[24] D. Ugarković and M. Plohl, "Variation in satellite DNA profiles_causes and effects," EMBO Journal, vol. 21, no. 22, pp. 5955-5959, 2002.

[25] C. I. Wu, T. W. Lyttle, M. L. Wu, and G. F. Lin, "Association between a satellite DNA sequence and the responder of segregation distorter in D. melanogaster," Cell, vol. 54, no. 2, pp. 179-189, 1988.

[26] R. Blattes, C. Monod, G. Susbielle et al., "Displacement of D1, HP1 and topoisomerase II from satellite heterochromatin by a specific polyamide," EMBO Journal, vol. 25, no. 11, pp. 2397-2408, 2006.

[27] J. H. Werren, "The paternal-sex-ratio chromosome of nasonia," The American Naturalist, vol. 137, pp. 392-402, 1991.

[28] D. G. Eickbush, T. H. Eickbush, and J. Werren, "Molecular characterization of repetitive DNA sequences from a B chromosome," Chromosoma, vol. 101, no. 9, pp. 575-583, 1992.

[29] J. J. Yunis and W. G. Yasmineh, "Heterochromatin, satellite DNA, and cell function," Science, vol. 174, no. 4015, pp. 1200-1209, 1971.

[30] J. L. Gerton and R. S. Hawley, "Homologous chromosome interactions in meiosis: diversity amidst conservation," $\mathrm{Na}$ ture Reviews Genetics, vol. 6, no. 6, pp. 477-487, 2005.

[31] S. Gershenson, "Studies on the genetically inert region of the $\mathrm{X}$ chromosome of Drosophila: I. Behavior of an X chromosome deficient for a part of the inert region," Journal of Genetics, vol. 28, pp. 297-312, 1933.

[32] B. D. McKee and D. L. Lindsley, "Inseparability of X heterochromatic functions responsible for X: Y pairing, meiotic drive and male fertility in Drosophila melanogaster males," Genetics, vol. 116, pp. 399-407, 1987.

[33] B. D. McKee and G. H. Karpen, "Drosophila ribosomal RNA genes function as an X-Y pairing site during male meiosis," Cell, vol. 61, no. 1, pp. 61-72, 1990.

[34] B. D. McKee, "Homologous pairing and chromosome dynamics in meiosis and mitosis," Biochimica et Biophysica Acta, vol. 1677, no. 1-3, pp. 165-180, 2004.

[35] R. S. Hawley, H. Irick, A. E. Zitron et al., "There are two mechanisms of achiasmate segregation in Drosophila females, one of which requires heterochromatic homology," Developmental Genetics, vol. 13, no. 6, pp. 440-467, 1993.

[36] M. Yamamoto, "Cytologic studies of heterochromatin function in the Drosophila melanogaster male: autosomal meiotic pairing," Chromosoma, vol. 72, no. 3, pp. 293-328, 1979.

[37] U. Tettenborn and A. Gropp, "Meiotic nondisjunction in mice and mouse hybrids," Cytogenetics, vol. 9, no. 4, pp. 272283, 1970.

[38] Y. Matsuda, P. B. Moens, and V. M. Chapman, "Deficiency of $\mathrm{X}$ and $\mathrm{Y}$ chromosomal pairing at meiotic prophase in spermatocytes of sterile interspecific hybrids between laboratory mice (Mus domesticus and Mus spretus)," Chromosoma, vol. 101, no. 8, pp. 483-492, 1992.

[39] G. L. Stebbins, "Cytogenetic studies in Paeonia II. The cytology of the diploid species and hybrids," Genetics, vol. 23, pp. 83-110, 1937.

[40] H. Hollocher, K. Agopian, J. Waterbury, R. W. O’Neill, and A. Davis, "Characterization of defects in adult germline development and oogenesis of sterile and rescued female hybrids in crosses between Drosophila simulans and Drosophila melanogaster," Molecular and Developmental Evolution, vol. 288, no. 3, pp. 205-218, 2000.

[41] D. A. Barbash and M. Ashburner, "A novel system of fertility rescue in Drosophila hybrids reveals a link between hybrid lethality and female sterility," Genetics, vol. 163, no. 1, pp. 217-226, 2003.

[42] C. W. Metz, "Chromosome studies on the Diptera: II. The paired association of chromosomes in the Diptera and its significance," Journal of Experimental Zoology, vol. 21, pp. 213279, 1919.

[43] S. Henikoff and L. Comai, "Trans-sensing effects: the ups-and downs of being together," Cell, vol. 93, no. 3, pp. 329-332, 1998.

[44] J. C. Fung, W. F. Marshall, A. Dernburg, D. A. Agard, and J. W. Sedat, "Homologous chromosome pairing in Drosophila melanogaster proceeds through multiple independent initiations," Journal of Cell Biology, vol. 141, no. 1, pp. 5-20, 1998.

[45] B. T. Sage and A. K. Csink, "Heterochromatic self-association, a determinant of nuclear organization, does not require sequence homology in Drosophila," Genetics, vol. 165, no. 3, pp. 1183-1193, 2003.

[46] R. G. Temin, "The independent distorting ability of the Enhancer of Segregation distortion, E(SD), in Drosophila melanogaster," Genetics, vol. 128, no. 2, pp. 339-356, 1991.

[47] K. Maeshima and U. K. Laemmli, "A Two-step scaffolding model for mitotic chromosome assembly," Developmental Cell, vol. 4, no. 4, pp. 467-480, 2003.

[48] J. W. Raff, R. Kellum, and B. Alberts, "The Drosophila GAGA transcription factor is associated with specific regions of heterochromatin throughout the cell cycle," EMBO Journal, vol. 13, no. 24, pp. 5977-5983, 1994. 
[49] T. Török, M. Gorjánácz, P. J. Bryant, and I. Kiss, "Prod is a novel DNA-binding protein that binds to the $1.686 \mathrm{~g} / \mathrm{cm}^{3} 10$ bp satellite repeat of Drosophila melanogaster," Nucleic Acids Research, vol. 28, no. 18, pp. 3551-3557, 2000.

[50] M. Z. Radic, K. Lundgren, and B. A. Hamkalo, "Curvature of mouse satellite DNA and condensation of heterochromatin," Cell, vol. 50, no. 7, pp. 1101-1108, 1987.

[51] M. Plohl, N. Meštrović, B. Bruvo, and D. Ugarković, "Similarity of structural features and evolution of satellite DNAs from Palorus subdepressus (Coleoptera) and related species," Journal of Molecular Evolution, vol. 46, no. 2, pp. 234-239, 1998.

[52] K. M. Bhat, G. Farkas, F. Karch, H. Gyurkovics, J. Gausz, and P. Schedl, "The GAGA factor is required in the early Drosophila embryo not only for transcriptional regulation but also for nuclear division," Development, vol. 122, no. 4, pp. 1113-1124, 1996.

[53] T. Nakayama, K. Nishioka, Y. X. Dong, T. Shimojima, and S. Hirose, "Drosophila GAGA factor directs histone H3.3 replacement that prevents the heterochromatin spreading," Genes and Development, vol. 21, no. 5, pp. 552-561, 2007.

[54] S. Henikoff, K. Ahmad, and H. S. Malik, "The centromere paradox: stable inheritance with rapidly evolving DNA," Science, vol. 293, no. 5532, pp. 1098-1102, 2001.

[55] C.-T. Ting, S. C. Tsaur, M.-L. Wu, and C.-I. Wu, "A rapidly evolving homeobox at the site of a hybrid sterility gene," Science, vol. 282, no. 5393, pp. 1501-1504, 1998.

[56] S. Sun, C. Ting, and C.-I. Wu, "The normal function of a speciation gene, Odysseus, and its hybrid sterility effect," Science, vol. 305 , no. 5680, pp. 81-83, 2004.

[57] C.-T. Ting, S.-C. Tsaur, S. Sun, W. E. Browne, Y.-C. Chen et al., "Gene duplication and speciation in Drosophila: evidence from the Odysseus locus," Proceedings of the National Academy of Sciences of the United States of America, vol. 101, no. 33, pp. 12232-12235, 2004.

[58] J. J. Bayes and H. S. Malik, "Altered heterochromatin binding by a hybrid sterility protein in Drosophila sibling species," Science, vol. 326, no. 5959, pp. 1538-1541, 2009.

[59] J. C. Eissenberg and G. Reuter, "Chapter 1 Cellular Mechanism for Targeting Heterochromatin Formation in Drosophila," International Review of Cell and Molecular Biology, vol. 273, pp. 1-47, 2009.

[60] M. Lachner, D. O'Carroll, S. Rea, K. Mechtler, and T. Jenuwein, "Methylation of histone H3 lysine 9 creates a binding site for HP1 proteins," Nature, vol. 410, no. 6824, pp. 116120, 2001.

[61] A. H. Peters, J. E. Mermoud, D. O'Carroll et al., "Histone H3 lysine 9 methylation is an epigenetic imprint of facultative heterochromatin," Nature Genetics, vol. 30, no. 1, pp. 77-80, 2002.

[62] T. Cheutin, A. J. McNairn, T. Jenuwein, D. M. Gilbert, P. B. Singh, and T. Misteli, "Maintenance of stable heterochromatin domains by dynamic HP1 binding," Science, vol. 299, no. 5607, pp. 721-725, 2003.

[63] P. J. Verschure, I. van der Kraan, W. de Leeuw et al., "In vivo HP1 targeting causes large-scale chromatin condensation and enhanced histone lysine methylation," Molecular and Cellular Biology, vol. 25, no. 11, pp. 4552-4564, 2005.

[64] G. Schotta, A. Ebert, V. Krauss et al., "Central role of Drosophila SU(VAR)3-9 in histone H3-K9 methylation and heterochromatic gene silencing," EMBO Journal, vol. 21, no. 5, pp. 1121-1131, 2002.
[65] L. Fanti and S. Pimpinelli, "HP1: a functionally multifaceted protein," Current Opinion in Genetics and Development, vol. 18, no. 2, pp. 169-174, 2008.

[66] K. Sawamura, M.-T. Yamamoto, and T. K. Watanabe, "Hybrid lethal systems in the Drosophila melanogaster species complex. II. The Zygotic hybrid rescue (Zhr) gene of Drosophila melanogaster," Genetics, vol. 133, no. 2, pp. 307-313, 1993.

[67] K. Sawamura and M.-T. Yamamoto, "Characterization of a reproductive isolation gene, zygotic hybrid rescue, of Drosophila melanogaster by using minichromosomes," Heredity, vol. 79, no. 1, pp. 97-103, 1997.

[68] K. Sawamura and M.-T. Yamamoto, "Cytogenetical localization of Zygotic hybrid rescue (Zhr), a Drosophila melanogaster gene that rescues interspecific hybrids from embryonic lethality," Molecular and General Genetics, vol. 239, no. 3, pp. 441449, 1993.

[69] K. Sawamura, A. Fujita, R. Yokoyama et al., "Molecular and genetic dissection of a reproductive isolation gene, zygotic hybrid rescue, of Drosophila melanogaster," Japanese Journal of Genetics, vol. 70, no. 2, pp. 223-232, 1995.

[70] D. A. Barbash, D. F. Siino, A. M. Tarone, and J. Roote, "A rapidly evolving MYB-related protein causes species isolation in Drosophila," Proceedings of the National Academy of Sciences of the United States of America, vol. 100, no. 9, pp. 53025307, 2003.

[71] D. C. Presgraves, L. Balagopalan, S. M. Abmayr, and H. A. Orr, "Adaptive evolution drives divergence of a hybrid inviability gene between two species of Drosophila," Nature, vol. 423, no. 6941, pp. 715-719, 2003.

[72] N. J. Brideau, H. A. Flores, J. Wang, S. Maheshwari, X. Wang, and D. A. Barbash, "Two Dobzhansky-Muller Genes interact to cause hybrid lethality in Drosophila," Science, vol. 314, no. 5803, pp. 1292-1295, 2006.

[73] J. C. Wang, "Cellular roles of DNA topoisomerases: a molecular perspective," Nature Reviews Molecular Cell Biology, vol. 3, no. 6, pp. 430-440, 2002.

[74] P. A. Coelho, J. Queiroz-Machado, and C. E. Sunkel, "Condensin-dependent localisation of topoisomerase II to an axial chromosomal structure is required for sister chromatid resolution during mitosis," Journal of Cell Science, vol. 116, no. 23, pp. 4763-4776, 2003.

[75] V. E. Foe, G. M. Odell, and B. A. Edgar, "Mitosis and morphogenesis in the Drosophila embryo: point and counterpoint," in The Development of Drosophila Melanogaster, M. Bate and A. Martinez Arias, Eds., pp. 149-300, Cold Spring Harbor Laboratory Press, New York, NY, USA, 1993.

[76] S. Pimpinelli, W. Sullivan, M. Prout, and L. Sandler, "On biological functions mapping to the heterochromatin of Drosophila melanogaster," Genetics, vol. 109, no. 4, pp. 701-724, 1985.

[77] R. Kellum, J. W. Raff, and B. M. Alberts, "Heterochromatin protein 1 distribution during development and during the cell cycle in Drosophila embryos," Journal of Cell Science, vol. 108, no. 4, pp. 1407-1418, 1995.

[78] O. Mihola, Z. Trachtulec, C. Vlcek, J. C. Schimenti, and J. Forejt, "A mouse speciation gene encodes a meiotic histone H3 methyltransferase," Science, vol. 323, no. 5912, pp. 373375, 2009.

[79] T. Rudolph, M. Yonezawa, S. Lein et al., "Heterochromatin formation in Drosophila is initiated through active removal of H3K4 methylation by the LSD1 homolog SU(VAR)3-3," Molecular Cell, vol. 26, no. 1, pp. 103-115, 2007. 
[80] D. Vermaak, S. Henikoff, and H. S. Malik, "Positive selection drives the evolution of rhino, a member of the heterochromatin protein 1 family in Drosophila," PLoS Genetics, vol. 1, no. 1, pp. 96-108, 2005.

[81] D. Moazed, "Small RNAs in transcriptional gene silencing and genome defence," Nature, vol. 457, no. 7228, pp. 413420, 2009.

[82] M. Pal-Bhadra, B. A. Leibovitch, S. G. Gandhi et al., "Heterochromatic silencing and HP1 localization in Drosophila are dependent on the RNAi machinery," Science, vol. 303, no. 5658, pp. 669-672, 2004.

[83] P. Fransz, R. ten Hoopen, and F. Tessadori, "Composition and formation of heterochromatin in Arabidopsis thaliana," Chromosome Research, vol. 14, no. 1, pp. 71-82, 2006.

[84] L. Usakin, J. Abad, V. V. Vagin, B. de Pablos, A. Villasante, and V. A. Gvozdev, "Transcription of the 1.688 satellite DNA family is under the control of RNA interference machinery in Drosophila melanogaster ovaries," Genetics, vol. 176, no. 2, pp. 1343-1349, 2007.

[85] L. Salvany, S. Aldaz, E. Corsetti, and N. Azpiazu, "A new role for hth in the early pre-blastodermic divisions in Drosophila," Cell Cycle, vol. 8, no. 17, pp. 2748-2755, 2009.

[86] P. M. Ferree and D. A. Barbash, "Distorted sex ratios: a window into RNA-mediated silencing," PLoS Biology, vol. 5, no. 11, article e303, 2007.

[87] D. U. Menon and V. H. Meller, "Germ line imprinting in Drosophila: epigenetics in search of function," Fly, vol. 4, no. 1, pp. 48-52, 2010.

[88] N. I. Noujdin, "The regularities of the heterochromatin influence on mosaicism. The hypothesis of the structural homozygosity and heterozygosity," Journal of General Biology, vol. 5, pp. 357-388, 1944.

[89] V. Lloyd, "Parental imprinting in Drosophila," Genetica, vol. 109, no. 1-2, pp. 35-44, 2000.

[90] K. G. Golic, M. M. Golic, and S. Pimpinelli, "Imprinted control of gene activity in Drosophila," Current Biology, vol. 8, no. 23, pp. 1273-1276, 1998.

[91] K. A. Maggert and K. G. Golic, "The Y chromosome of Drosophila melanogaster exhibits chromosome-wide imprinting," Genetics, vol. 162, no. 3, pp. 1245-1258, 2002.

[92] C. Merrill, L. Bayraktaroglu, A. Kusano, and B. Ganetzky, "Truncated RanGAP encoded by the segregation distorter locus of Drosophila," Science, vol. 283, no. 5408, pp. 1742 $1745,1999$.

[93] A. Kusano, C. Staber, and B. Ganetzky, "Nuclear mislocalization of enzymatically active RanGAP causes segregation distortion in Drosophila," Developmental Cell, vol. 1, no. 3, pp. 351-361, 2001.

[94] Y. Tao, L. Araripe, S. B. Kingan, Y. Ke, H. Xiao, and D. L. Hartl, "A sex-ratio meiotic drive system in Drosophila simulans II: an X-linked distorter," PLoS biology, vol. 5, no. 11, article e293, 2007.

[95] N. Phadnis and H. A. Orr, "A single gene causes both male sterility and segregation distortion in Drosophila hybrids," Science, vol. 323, no. 5912, pp. 376-379, 2009.

[96] E. Novitski and L. Sandler, "Are all products of spermatogenesis regularly functional?" Proceedings of the National Academy of Sciences of the United States of America, vol. 43, pp. 318-324-318-324, 1957.

[97] M. E. Zwick, J. L. Salstrom, and C. H. Langley, "Genetic variation in rates of nondisjunction: association of two naturally occuring polymorphisms in the chromokinesin nod with increased rates of nondisjunction in Drosophila melanogaster," Genetics, vol. 152, no. 4, pp. 1605-1614, 1999.
[98] H. S. Malik, "The centromere-drive hypothesis: a simple basis for centromere complexity," Progress in Molecular and Subcellular Biology, vol. 48, pp. 33-52, 2009.

[99] S. I. Agulnik, A. I. Agulnik, and A. O. Ruvinsky, "Meiotic drive in female mice heterozygous for the HSR inserts on chromosome 1," Genetical Research, vol. 55, no. 2, pp. 97$100,1990$.

[100] E. S. Buckler, T. L. Phelps-Durr, C. S. Buckler, R. K. Dawe, J. F. Doebley, and T. P. Holtsford, "Meiotic drive of chromosomal knobs reshaped the maize genome," Genetics, vol. 153, no. 1, pp. 415-426, 1999.

[101] J. Jaenike, "Sex chromosome meiotic drive," Annual Review of Ecology and Systematics, vol. 32, pp. 25-49, 2001.

[102] L. Fishman and J. H. Willis, "A novel meiotic drive locus almost completely distorts segregation in Mimulus (monkeyflower) hybrids," Genetics, vol. 169, no. 1, pp. 347-353, 2005.

[103] L. Fishman and A. Saunders, "Centromere-associated female meiotic drive entails male fitness costs in monkeyflowers," Science, vol. 322, no. 5907, pp. 1559-1562, 2008.

[104] H. S. Malik and J. J. Bayes, "Genetic conflicts during meiosis and the evolutionary origins of centromere complexity," Biochemical Society Transactions, vol. 34, no. 4, pp. 569-573, 2006.

[105] H. A. Orr, "Dobzhansky, Bateson, and the genetics of speciation," Genetics, vol. 144, no. 4, pp. 1331-1335, 1996.

[106] N. A. Johnson, "Hybrid incompatibility genes: remnants of a genomic battlefield?" Trends in Genetics, vol. 26, no. 7, pp. 317-325, 2010.

[107] D. A. Barbash and J. G. Lorigan, "Lethality in Drosophila melanogaster/Drosophila simulans species hybrids is not associated with substantial transcriptional misregulation," Journal of Experimental Zoology Part B: Molecular and Developmental Evolution, vol. 308, no. 1, pp. 74-84, 2007.

[108] D. Ortíz-Barrientos, B. A. Counterman, and M. A. F. Noor, "Gene expression divergence and the origin of hybrid dysfunctions," Genetica, vol. 129, no. 1, pp. 71-78, 2007.

[109] H. A. Orr, "The population genetics of speciation: the evolution of hybrid incompatibilities," Genetics, vol. 139, no. 4, pp. 1805-1813, 1995. 

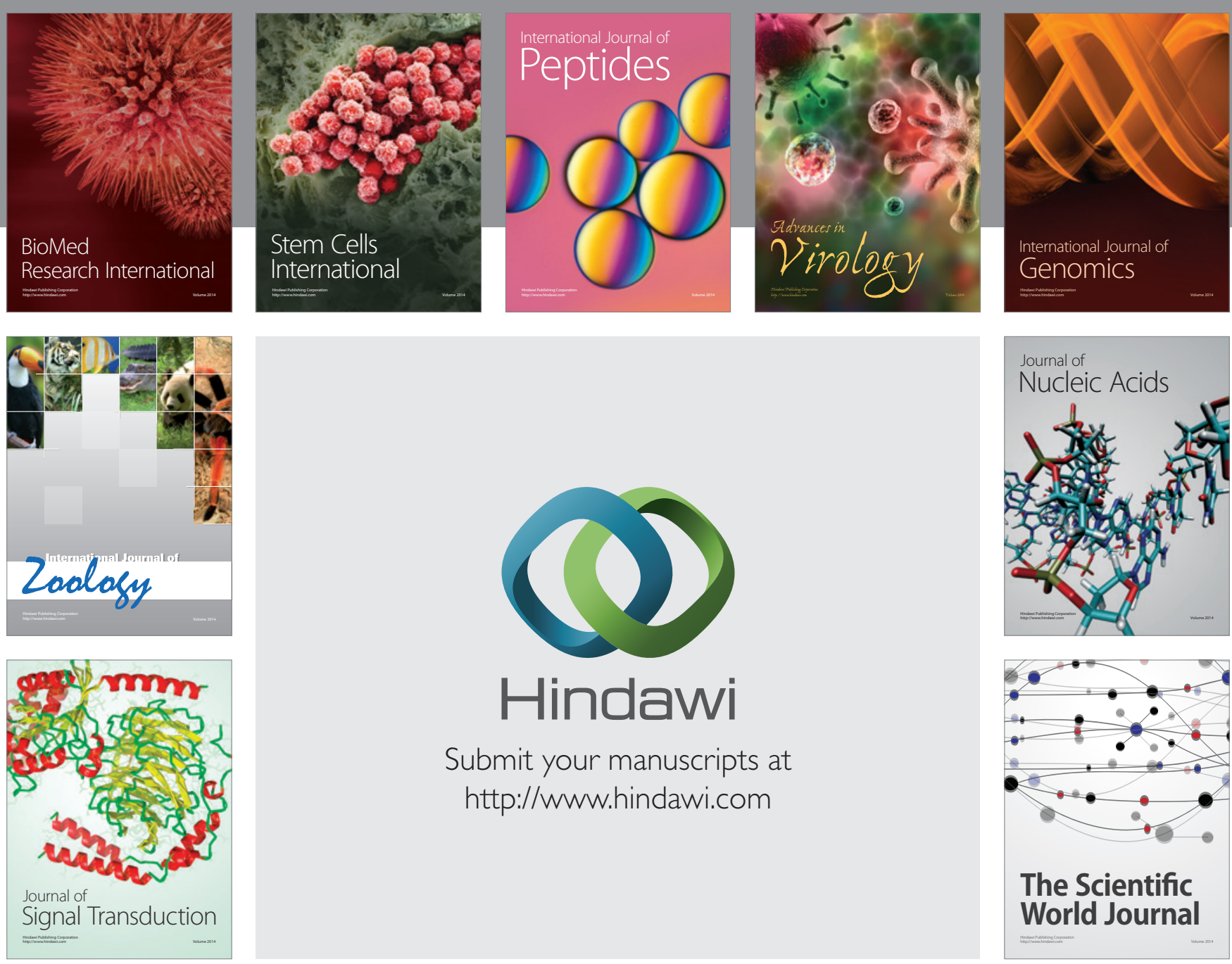

Submit your manuscripts at

http://www.hindawi.com
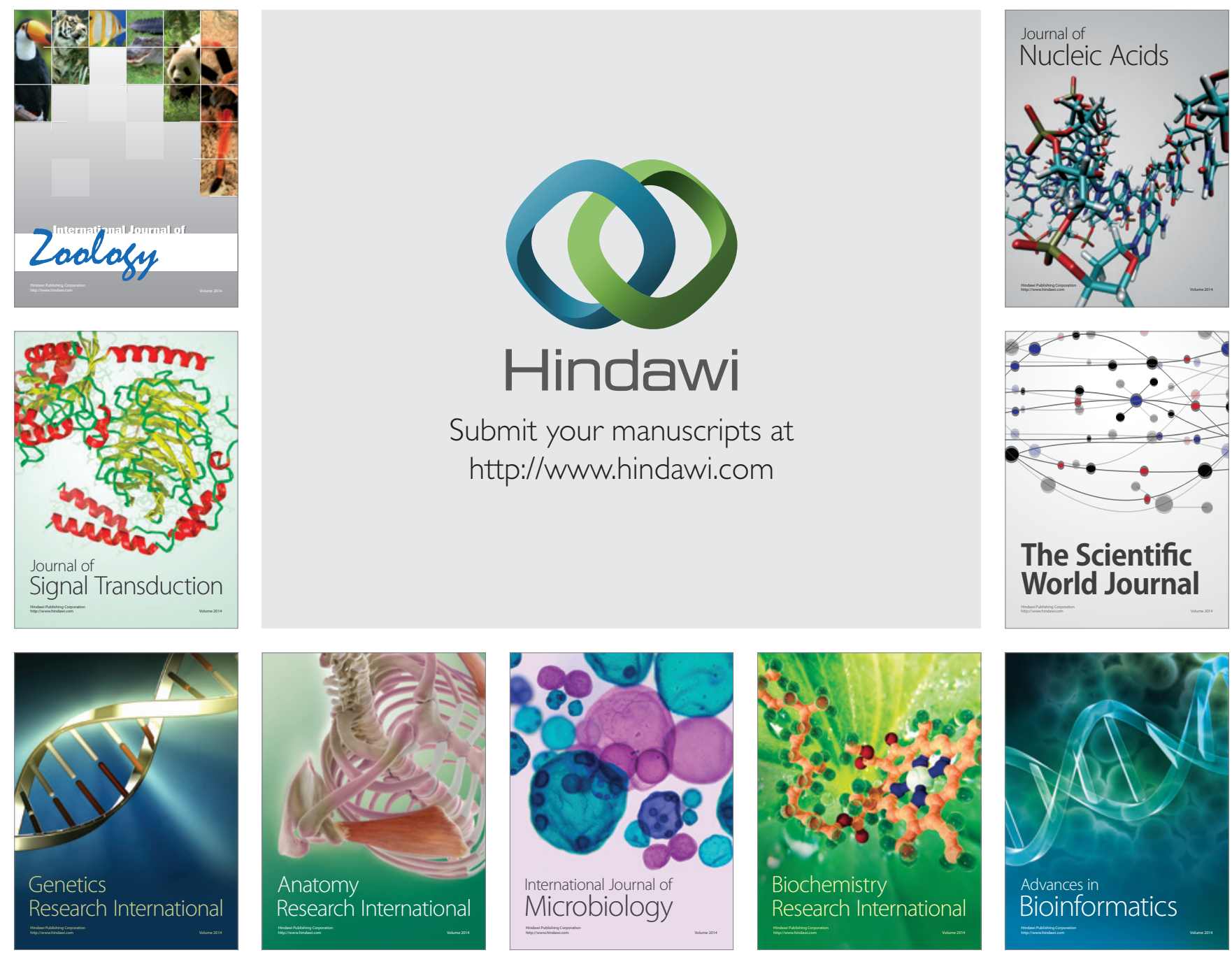

The Scientific World Journal
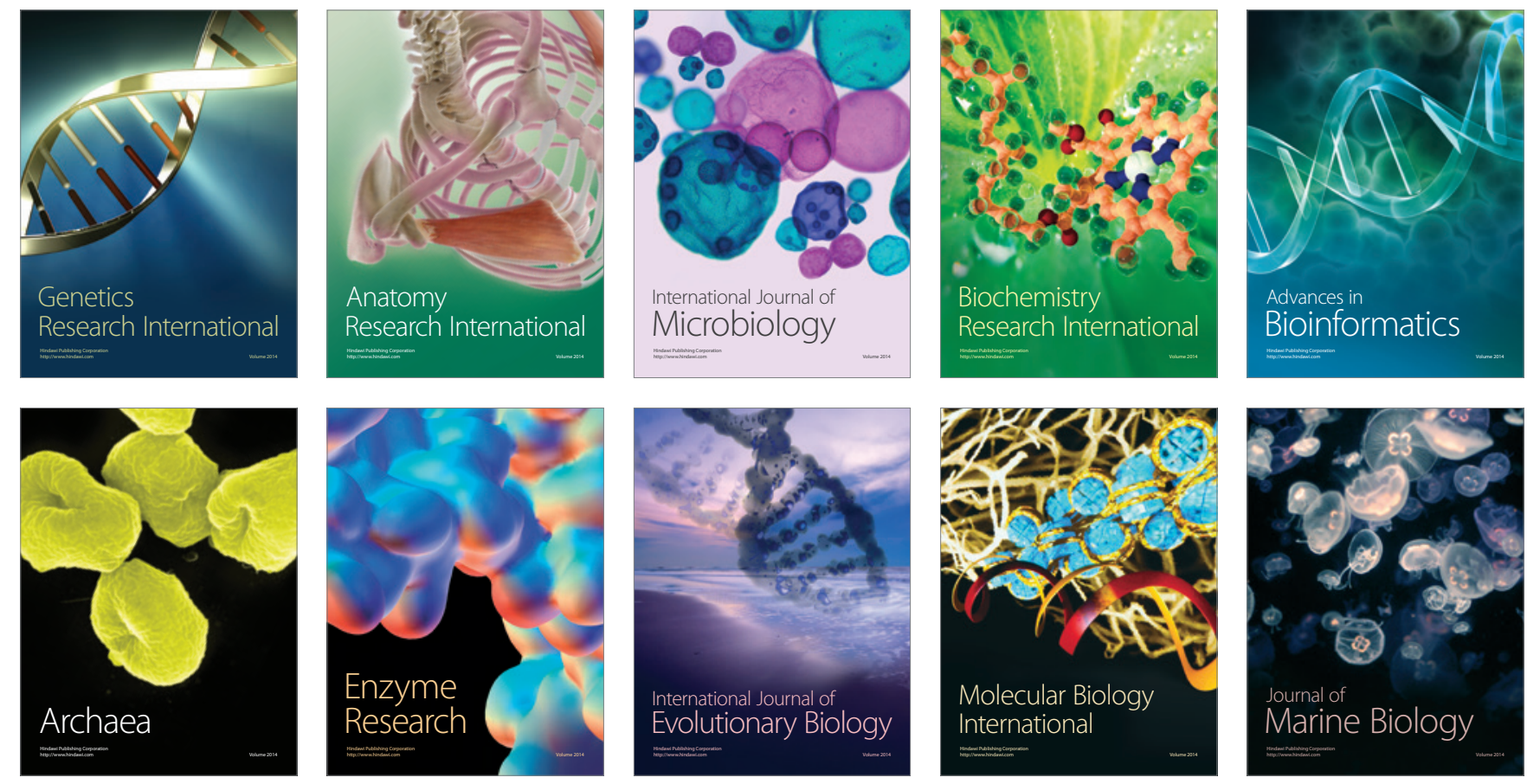